\title{
Propuesta metodológica para la transversalización de los Derechos Sexuales Reproductivos desde la Práctica Pedagógica
}

\author{
Methodological proposal for the mainstreaming of the sexual and reproductive rights \\ seen from a pedagogical practice perspective \\ Proposta metodológica para a transversalização dos Direitos Sexuais Reprodutivos \\ desde a Prática Pedagógica
}

DOI: http://dx.doi.org/10.21803\%2Fpenamer.11.21.537

Judith Martinez Royert ORCID: 0000-0002-4468-8578

Felix Parra Dominguez ORCD: 0000.0002.0259.8160

Mary Rodríguez Pérez https://orcid.org/0000-0002-8263-3412

Jacqueline Rosa Cervantes Schiller

\section{¿Cómo citar este artículo?}

Martinez, J., Parra, F., Rodríguez, M., Cervantes, J., (2018). Propuesta metodológica para la transversalización de los Derechos Sexuales Reproductivos desde la Práctica Pedagógica. Pensamiento Americano, 11(21), 237-252

DOI:http://dx.doi.org/10.21803\%2Fpenamer.11.21.537

\begin{abstract}
Resumen
El propósito de este artículo fue diseñar una propuesta metodológica para la tansverzalición de los Derechos Sexuales y Reproductivos desde la práctica pedagógica, a partir de una caracterización de las concepciones de los maestros sobre el tema y la identificación de las acciones de promoción de los D.S.R. desarrolladas por ellos, lo que permitió establecer los elementos de la propuesta metodológica. El camino metodológico se asumió desde un enfoque cualitativo descriptivo, y de investigación acción participativa, con la totalidad de la población docente (10), a quienes se les aplicó una entrevista abierta; se realizó análisis textual discursivo; como apoyo al proceso se trabajó con grupo focal. Entre los hallazgos y conclusiones más relevantes se encuentran: las actividades desarrolladas por los maestros en relación con la promoción de los D.S.R., son pocas, y aisladas del ejercicio de su práctica pedagógica. Los D.S.R., deben asumirse desde una perspectiva transversal en el currículo; se considera fundamental la cualificación de los maestros y buscar alternativas que conlleven a involucrar a los padres y madres de familia en estos procesos, con el objetivo que todos los actores del sistema educativo se comprometan de forma decidida.
\end{abstract}

PALABRAS CLAVE: Derechos Reproductivos, Práctica pedagógica, Profesores, niño; Educación

\begin{abstract}
This article is the outcome of a research whose purpose was to design a methodological proposal for the sexual and reproductives rights mainstreaming seen from the pedagogical practice perspective. Starting with a characterization of the teachers concepts about the proposal and the identification of the actions promoting the SRR developed by themselves permitted us to establish the elements composing the methodological proposal. The methodology used was qualitive-descriptive and a participative research including the whole amount of the teacher population (10) who underwent an open interview,and a textual speech analysis as a support, the process was focused on a determined group. Amongst the findings and more relevant conclusions, we discovered that the actions developed by teachers in terms of the SRR promotion are very few and isolated from real pedagogical practice. The SRR must be assumed from a mainstreaming perspective in the curricula;it is considered essential the teachers qualifications and look for alternatives that lead to more parents involvement in this processes, aiming at a total and decisive commitment of all the educative system agents.
\end{abstract}

KEYWORDS : Reproductive rights, pedagogical practice, teachers, child, education 


\section{Resumo:}

Este artigo é resultado de uma pesquisa cujo propósito foi desenhar uma proposta metodológica para a transversalização dos Direitos Sexuais e Reprodutivos desde a prática pedagógica, a partir de uma caracterização das concepções dos mestres professores sobre o tema e a identificação das ações de promoção dos D.S.R. desenvolvidas por eles, o que permitiu estabelecer os elementos da proposta metodológica. O caminho metodológico foi assumido desde um enfoque qualitativo descritivo, e de pesquisa de ação participativa, com a totalidade da população docente (10) aos quais foram aplicadas entrevistas abertas; foi realizada a análise textual discursiva; como apoio ao processo se trabalhou com grupo focal. Entre os descobrimentos e conclusões mais importantes se encontram: as atividades desenvolvidas pelos professores em relação à promoção dos D.S.R., são poucas, e afastadas do exercício de sua prática pedagógica. Os D.S.R. devem assumir-se desde uma perspectiva transversal no currículo; se considera fundamental a qualificação dos professores e buscar alternativas que levem ao envolvimento dos pais e mães de famílias nesses processos, com o objetivo de que todos os atores do sistema educativo se comprometam de forma decidida.

PALAVRAS CHAVE: Direitos Reprodutivos, Prática pedagógica, Professores, criança; Educação

\section{Perfil}

PhD en Ciencias de la Educación de la Universidad del Atlántico; Magister en educación con énfasis en cognición de la Universidad del Norte; Grupo RELEDUC-Universidad simón Bolivar

Jmartinezroyert@gmail.com

\section{Perfil}

Magister en Educación, Especialista en Docencia; Institución Educativa Normal Superior de Sincelejo.

felixparra@ustadistancia.edu.co

\section{Perfil}

Especialista en Docencia Universidad de la Costa

\section{Perfil}

Especialista en Docencia; Corporación Unificada Nacional CUN

Judith Martinez Royert Enfermera

Felix Parra Dominguez

Licenciado en

psicopedagogía

Mary Rodríguez Pérez Pedagoga

Jacqueline Rosa Cervantes Schiller

Pedagoga 


\section{Introducción}

E

n Colombia en las últimas décadas se ha venido planteando la necesidad de fortalecer desde el interior de las Instituciones Educativas la formación de los niñas, niños, jóvenes y adolescentes en el campo de la sexualidad y la construcción de ciudadanía; contemplando de esta forma como eje principal el fortalecimiento y vivencias de los derechos humanos sexuales y reproductivos.

Atendiendo a lo anterior han surgidos distintas iniciativas entre las cuales se pueden relacionar las siguientes: las guías pedagógicas para la convivencia escolar soportadas en la ley 1620 del 2013 y el decreto1965 del mismo año y el programa de educación para la sexualidad y construcción de ciudadanía que establece desde la concepción universal del ser humano los componentes y funciones con miras al ejercicio plenoy sano de la sexualidad humana.

Lo anterior, pone en evidencia que en el país existe una política de educación para la sexualidad encaminada hacia el ejercicio de los derechos humanos sexuales y reproductivos (DHSR), la formación de personas con una capacidad reflexiva y critica para manejar adecuadamente las situaciones en relación consigo misma, con los demás y con su entorno, mediante el ejercicio pleno de los derechos sexuales y reproductivos (DSR) además, se desarrollen como ciudadanos. Por consiguiente, la política pública de educación para la sexualidad y construcción de ciudadanía en Colombia propone como reto la promoción de conocimientos, habilidades, actitudes, valores y comportamientos que fortalezcan la dignidad humana y el ejercicio de los derechos, en particular los relacionados con sexuales y reproductivos.

En consecuencia, la formación de los ciudada- nos para la democracia sexual debe estar encaminada hacia un profundo conocimiento y sometimiento de los derechos como regla máxima de convivencia humana. Esto implica que debe existir un trascendental respeto por los estilos de vida sexual, vida reproductiva de pareja y de género (Velis, 2017); (Giraldo, 2006).

Por tal motivo, la escuela debe propiciar el respeto a la vida, el fin de la violencia, la promoción y práctica de la no violencia por medio de la educación, el diálogo y la cooperación (Rodríguez, K. \& Hinojosa, M. (2017) además, se debe convertir en un espacio donde se manifiesten las particularidades de cada individuo, con el propósito de identificarla, estimarlas, familiarizarse con ellas e incluirlas; lo que denota que la formación en la sexualidad, contribuye a la generación de ambientes propicios para el desarrollo de competencia y conocimiento donde los individuos de la sociedad sean personas que luchen por el respeto y libertad de sus derechos sexuales y reproductivos (DSR), no solo para que se cumpla en su ser individual, sino en el colectivo de la comunidad (Rodríguez-Burgos y García, 2017).

La práctica pedagógica de los maestros de hoy debe estar encaminada al desarrollo de competencias en los estudiantes para que estos puedan incorporar, en su cotidianidad, el ejercicio de los derechos sexuales y reproductivos (DSR) y tomen decisiones que le permitan vivir una sexualidad plena, sana y responsable además de enriquecer su proyecto de vida y el de los demás.

La investigación partió del análisis de las concepciones de los docentes frente a los DSR, esto llevó a una caracterización de ellas sobre la base de la integralidad existente entre la sexualidad y la reproducción con el sujeto y la sociedad y finalmente se diseñó una propuesta para articular las prácticas pedagógicas, los saberes disciplinar de cada una de las áreas 
con elementos relacionados con la sexualidad y la reproducción, todo desde un enfoque transversal.

Este trabajo de investigación permitió que los actores involucrados generaran en primera instancia conciencia crítica sobre la importancia de abordar los DSR como parte fundamental en la formación del ser humano; en segunda ayudó a que el maestro en el ejercicio realizara múltiples búsquedas de estrategias metodológicas, recursos y estrategias pedagógicas con mira a la transformación de sus prácticas pedagógicas, en tercera medida la implementación de una nueva práctica por parte de maestros en ejercicio conlleva a que se dé un proceso efectivo de mediación entre los diferentes actores del sistema educativo generando transformaciones desde las actitudes, la participación y la convivencia entre estudiantes.

El desarrollo de estudio permitió establecer de qué forma los maestros conciben los D.S.R como parte del abordaje transversal de su práctica pedagógica; de esta manera a partir del proceso de investigación se suscitó la reflexión crítica en los maestros, desde su discurso, conocimientos e implementación de estrategias para la articulación de los D.S.R.

Teniendo en cuenta los elementos planteados anteriormente se consideró abordar esta problemática en una Institución Educativa Rural, con el objetivo de explorar en los maestros el estado de empoderamiento frente a los D.S.R , además, si estos son abordados en la práctica pedagógica, desde otra perspectiva en términos de convivencia escolar y manifestaciones de actitudes entre estudiantes, entre docentes-estudiantes $\mathrm{y}$, si se hace visible desde el lenguaje y las expresiones de afecto; igualmente, valorar los derechos violentos que ocasionan conflictos entre los autores de la comunidad educativa; frente a lo anterior se puede decir que todos estos aspectos relacionados con la sexualidad y la reproducción humana al interior de la Institución Educativa pasan directamente a manos del comité de convivencia, ratificando de esta forma, una participación del maestro, nula o despectiva, en la resolución de problemáticas de esta índole, por consiguiente se planteó la siguiente pregunta de investigación; ¿Cuáles son las concepciones del maestro de básica primaria sobre los derechos sexuales y reproductivos desde el ejercicio de la práctica pedagógica en la I.E Rural?.

\subsection{Concepciones de Maestros}

En términos de la formación de maestros es fundamental explorar de manera permanente cuál es el tipo de conocimiento compartido con los estudiantes en el aula de clases; Se entiende por aula el espacio educativo en el que todas las personas que lo ocupan desarrollan papeles de agente activo en la construcción de normas, en la reelaboración de las normas sociales, de los valores y en la construcción de reglas de relación social (González citado por González, Vásquez, Campo, Miranda, Silvera Sarmiento, Agudelo \& Parra 2014). En ella, es fundamental hacer un monitoreo con el propósito de evaluar la efectividad, coherencia e impacto de estos en los comportamientos de los estudiantes; sin duda alguna se hace necesario expresar en términos de concepciones si estos conocimientos son debidamente mediados o no y si las estrategias que se definen para dicha mediación son coherentes al saber y a las necesidades de los estudiantes.

Para el presente trabajo investigativo se hizo una exploración en los maestros acerca de los derechos humanos sexuales y reproductivos (D.H.S.R) y de qué forma estas se reflejan en sus prácticas pedagógicas; frente a ello es fundamental que el quehacer docente se transforme de manera permanente teniendo en cuenta la competencias específicas de las 
áreas fundamentales pero también buscar mecanismos para incorporar aquellos temas que están relacionados con la formación humana y se vea reflejado desde las creencias, constructos, teorías implícitas, entre otras.

Las concepciones corresponden a las representaciones mentales que posee una persona para dar explicación y fundamentar una acción o comportamiento en una situación particular. A partir de ellas la persona analiza y construye una realidad para comprenderla y ser capaz de resolver situaciones presentes o dar explicaciones de la misma e integrarla a su entorno (Giordan, (1996). Las creencias del profesor se convierten en factor relevante en la construcción de la realidad y del aprendizaje (Pecharromán, 2004 citado en Basto-Torrado, 2011).

\subsection{Practicas Pedagógicas}

En la práctica docente debe considerarse la práctica pedagógica investigativa, también es considerada como una práctica social en donde intervienen múltiples subjetividades y juegos del lenguaje, por eso, la práctica pedagógica constituye un tipo de acción social específico, sobre la cual el maestro debe reflexionar individual y colectivamente de manera permanente para convertirla en una verdadera praxis pedagógica Santos, Baquero, Molano \& Pardo (2006). También, ha de entenderse como un proceso autónomo en el que confluyen las dinámicas formativo/ académicas correspondientes a la licenciatura: más que un requisito de orden formal ha de tomarse como un escenario, en donde se demarcan los compromisos del ser, quehacer y saber pedagógicos (Vásquez, 2006). Desde esta perspectiva la práctica docente trae consigo la reflexión, resignificación del quehacer docente en el aula, a partir de la necesidad de formación integral donde se conjuguen los saberes disciplinares y de formación ética y humana.
Las prácticas pedagógicas están fundamentadas en aprendizajes adquiridos durante toda la formación educacional recibida por el profesional docente desde su niñez, esto en complemento a la existencia de representaciones acerca de lo que es ser un buen profesor. Los docentes tienden a contextualizar sus prácticas en relación con lo vivenciado por ellos mismos como estudiantes (Cortez, Fuentes, Villablanca \& Guzmán, 2013).

\subsection{Derechos Sexuales y Reproductivos}

La salud sexual y reproductiva es fundamental para las personas, parejas y familias, así como para el desarrollo social y económico de las comunidades y naciones (Izquierdo, 2013). "La salud sexual y reproductiva (SSR) se refiere a un estado general de bienestar físico, mental y social, y no a la mera ausencia de enfermedades o dolencias en todos los aspectos relacionados con la sexualidad y reproducción, y entraña la posibilidad de ejercer los derechos sexuales y reproductivos Ministerio de Protección Social" (2003, P 9).

La SSR trae consigo el deleite de una vida sexual plena y satisfactoria, el derecho a procrear, decidir el número de hijos que desea tener, tomar decisiones en su sexualidad sin recibir presión o sin que se ejerza poder coercitivo para ello, tener la posibilidad de seleccionar el método de planificación familiar; un buen estado de salud sexual también implica erradicar toda clase de violencia doméstica y sexual, mantener una integridad física y mental , tener acceso a los servicios de salud, consejería en lo relacionado con el tema, es decir, satisfacer las necesidades específicas a la persona según su ciclo vital (Ríos, 2008)

Además, la educación sexual, es una herramienta para contribuir al cumplimiento de los DSR porque brinda a los individuos, en especial a los adolescentes, los conocimientos necesarios para tomar decisiones responsables 
en su sexualidad (Preinfalk-Fernández, 2015).

Lo anterior, es compromiso de todos, incluye la participación de varios sectores que actúen de manera articulada, en donde la escuela, se comprometa a desarrollar actividades curriculares y extracurriculares para disminuir las carencias en el conocimiento y abordaje por parte de profesores y estudiantes; para ello debe tenerse en cuenta la cultura, niveles educativos y el empoderamiento de los participantes frente al tema, de esta manera, garantizar una intervención clara, efectiva que satisfaga las necesidades de la población intervenida. (Ramírez Durán, G., \& Barriento García, M., 2015). La sensibilización de los actores educativos, en temas relacionados con la SSR se convierte en una idea fuerza para lograr el reconocimiento y respeto de los DSR en las escuelas; para ello se considera fundamental el trabajo colaborativo de las Instituciones (gobierno, escuela y familia) donde se asuman posturas críticas y responsables en relación con los derechos de la salud sexual y reproductiva de nuestros niños, jóvenes y adolescentes.

\section{Metodología:}

El presente estudio buscó diseñar una propuesta metodológica para el abordaje transversal de los Derechos Sexuales y Reproductivos (DSR) desde la práctica pedagógica en la I.E rural de un municipio de la costa norte colombiana, a partir de una caracterización de las concepciones sobre los D.S.R de los maestros de básica primaria y la identificación de las acciones de promoción de los D.S.R desarrolladas por los maestros, ello permitió establecer los elementos de la propuesta metodológica.

La construcción de la propuesta, se asumió un enfoque cualitativo descriptivo, teniendo en cuenta como parámetro fundamental la investigación acción participativa (IAP), el cual es una metodología que apunta a la producción de un conocimiento propositivo y trasfor- mador, se trabajó con el total de la población 10 docentes dado a que es una escuela, pequeña ubicada en la zona rural de un Departamento costero, participaron de manera voluntaria y se les aplicó una entrevista abierta (en la Institución Educativa fuera de jornada laboral, bajo condiciones ambientales adecuadas); Con la información obtenida de las entrevistas a docentes, se realizó el abordaje cualitativo de los datos con enfoque de análisis textual discursivo según lo plantea Moraes y Galiazzi, (2007); se realizó el proceso de unitarización o fragmentación, categorización y el respectivo metatexto. Igualmente, como apoyo al proceso se trabajó con la metodología de grupo focal para ampliar aspectos relacionados con las concepciones y las prácticas pedagógicas respetando la confidencialidad de cada uno de los participantes. En el procesamiento de la información se y análisis se tuvo presente el análisis interactivo, es decir se hizo lectura nuevamente de los datos con los informantes (profesores) para asegurar la validez de los hallazgos, lo cual permitió verificar la congruencia de las interpretaciones de los investigadores con los actores o docentes.

\section{Resultados y Discusión}

\subsection{Análisis o Discusión de Resultados}

Para el bordaje cualitativo de la información se realizó una lectura de las respuestas de cada pregunta, seguido de unitarización o fragmentación, categorización, desde las unidades hasta las categorías finales.

\section{Caracterización de las Concepciones sobre los D.S.R.}

A continuación, se presentan las concepciones que tienen los docentes acerca de los derechos sexualesy reproductivos de la Institución Educativa Rural. Se utilizó el abordaje cualitativo de los datos con enfoque de análisis textual discursivo Moraes \& Galiazzi, (2007); los docentes participantes respondieron cinco preguntas 
abiertas relacionadas con concepciones, consecuencias de la falta de conocimiento acerca del tema en los estudiantes, practica pedagógica, la promoción de los DSR y su transversalidad.

Inicialmente se realizó una lectura de las respuestas de cada pregunta, seguido de una unitarización o fragmentación como Tabla 1 .

Unidades de las categorías finales. lo orientan Moraes \& Galiazzi (2007), luego de este paso se hizo la categorización propiamente dicha, desde las unidades hasta las categorías finales como se muestra en la tabla 1.

Un aspecto importante del proceso de formación de un niño es darle a conocer cuáles son

\section{CATEGORIAS FINALES CATEGORIAS INIERMEDIAS CATEGORIAS IIICLALES UNIDADES}

\section{¿Qué piensa usted sobre los DSR?}

\begin{tabular}{|l|l|l|l|}
\hline Concepciones & Definición & Toma de Decisiones & $\begin{array}{l}\text { Construcción del provecto sexual, de ciudadanía en la } \\
\text { institución, decidir sobre la sexualidad, identidad de } \\
\text { género, autoestima, libertad }\end{array}$ \\
\hline & Función & & $\begin{array}{l}\text { Cuidado de su cuerpo y del respeto, } \\
\text { proteger la libertad y la autoestima, decidir cuál es su } \\
\text { sexualidad. }\end{array}$ \\
\hline ¿Qué efectos puede tener para los estudiantes no conocer cuáles son sus DSR? & $\begin{array}{l}\text { Abandono de los estudios por embarazos. Se limita su } \\
\text { proceso educativo }\end{array}$ \\
\hline Vida Sexual & Vulnerabilidad & Aislamiento & $\begin{array}{l}\text { El desconocimiento de los D.S.R hace que los niños sufran } \\
\text { más, se le coarta su libertad, no pueden decidir }\end{array}$ \\
\hline & Socialización & Indefensión & $\begin{array}{l}\text { No pueden elegir cómo cuidar su cuerpo, no se desarrollan } \\
\text { en su parte social }\end{array}$ \\
\hline & Sexualidad & Inseguridad & $\begin{array}{l}\text { aspecto fundamental del desarrollo y de los derechos } \\
\text { humanos }\end{array}$ \\
\hline & Proceso & $\begin{array}{l}\text { necesidades especificas de acuerdo con su ciclo vital, } \\
\text { aumentan nivel de pobreza, desarrollan infecciones de } \\
\text { transmisión sexual }\end{array}$ \\
\hline
\end{tabular}

¿Desde tu perspectiva como maestra explica qué tipo de mecanismo puedes utilizar para abordar temas relacionados con los temas de D.S.R en tu practica pedagógica?

\begin{tabular}{|c|c|c|c|}
\hline \multirow[t]{4}{*}{ Practica Pedagógica } & Conocimientos & Información & $\begin{array}{l}\text { Información sobre la salud sexual. reconocimiento del } \\
\text { cuerpo, respeto del cuerpo. }\end{array}$ \\
\hline & & Enunciados & $\begin{array}{l}\text { El maestro debe reflexionar individual y colectivamente } \\
\text { sobre aspectos de la sexualidad con los niños }\end{array}$ \\
\hline & Estrategias didácticas & & $\begin{array}{l}\text { Talleres, provectos, investigaciones, provectos pedagógicos } \\
\text { de aula, mesa redonda, charlas educativas, dibujo, escritos. }\end{array}$ \\
\hline & Trabajo interdisciplinario & & $\begin{array}{l}\text { Involucrar al equipo de bienestar en el acompañamiento } \\
\text { del estudiante, }\end{array}$ \\
\hline \multicolumn{4}{|c|}{ ¿En su comunidad educativa que acciones de promoción de los DSR implementas para garantizar que se lleve a cabo una sexualidad sana y responsable? } \\
\hline \multirow[t]{3}{*}{ Acciones de promoción } & $\begin{array}{l}\text { Diagnóstico de necesidades según } \\
\text { los problemas desde los estudiantes }\end{array}$ & & $\begin{array}{l}\text { Identificar si existe el respeto por las diferencias, desarrollo } \\
\text { de la personalidad, igualdad, }\end{array}$ \\
\hline & $\begin{array}{l}\text { Capacitación a los docentes } \\
\text { a partir de un diagnóstico de } \\
\text { necesidad educativas. }\end{array}$ & & $\begin{array}{l}\text { Manejar conceptos amplios acerca del tema y cómo } \\
\text { abordarlo con los estudiantes }\end{array}$ \\
\hline & $\begin{array}{l}\text { Jornada de sensibilización sobre } \\
\text { DSR en cada asignatura }\end{array}$ & & $\begin{array}{l}\text { Los estudiantes deben conocer los DSR para llevar a cabo una } \\
\text { sexualidad responsable y debe hacerse en las asignaturas }\end{array}$ \\
\hline \multicolumn{4}{|c|}{ ¿Qué elementos se deben tener en cuenta para involucrar los DSR dentro de tu práctica pedagógica como eje transversal? } \\
\hline \multirow[t]{2}{*}{ Transversalidad } & & Carácter interdisciplinario & $\begin{array}{l}\text { Crear condiciones favorables para proporcionar a los } \\
\text { alumnos mejor formación. } \\
\text { Unificar los estándares }\end{array}$ \\
\hline & $\begin{array}{l}\text { Proyectos pedagógicos } \\
\text { de aulas transversales }\end{array}$ & & $\begin{array}{l}\text { Provectos pedagógicos de aula donde participen todas las } \\
\text { asignaturas y en todos los niveles de formación. }\end{array}$ \\
\hline
\end{tabular}


sus derechos, entre ellos los D.S.R.; en esta dirección, las respuestas dadas por los docentes de básica primaria la Institución Educativa Rural ante las diferentes preguntas acerca del concepto personal que tienen cada uno de ellos, así como concepciones, vida sexual, practica pedagógica, diagnóstico y transversalidad de los D.S.R , ofrecen diversas perspectivas acerca de sus concepciones que servirá para comprender de qué manera ellos involucran esta temática en el proceso formativo de los estudiantes.

Dado el alcance, a continuación, se abordan solo las categorías finales: concepciones, vida sexual, práctica pedagógica, diagnóstico y transversalidad.

\section{Concepciones}

Cuando se hace referencia a la formación de maestros es fundamental que el profesional esté en permanente formación para que brinde un conocimiento eficaz a los estudiantes en el aula de clases, es primordial hacer un monitoreo que permita evaluar la efectividad, coherencia e impacto de estos en los comportamiento individual y social de los estudiantes; se hace necesario entonces, expresar en términos de concepciones si estos conocimientos son debidamente mediados o no y si las estrategias que se definen para dicha mediación son coherentes al saber y a las necesidades de los estudiantes.

El concepto de concepción posee una serie de términos con significados similares como: creencias, constructos, teorías implícitas, conocimiento práctico; tales términos están asociados a las teorías de la acción, de los constructos personales. Los docentes que imparten sus conocimientos en relación con D.S.R lo hacen bajo sus criterios, algunos maestros expresan su concepción sobre sexualidad, desde su práctica pedagógica, de la siguiente manera: "desde el aula de clase particularmente lo manejo hablándole a los niños y a las niñas acerca del cuidado de su cuerpo y del respeto que se deben tener con sus compañeros". Para ellos educar en D.S.R se trata se cuidado del cuerpo; ello confirma lo que manifiesta García \& González (2017), cuando asevera que alrededor del concepto de la sexualidad circula un sobre saber de la misma, relacionadas con la higienización, entendida como el dispositivo que regula los saberes y prácticas de sí mismo, donde el cuerpo es un aspecto importante para moldear, se le asigna la educación de éste, la instrucción desde lo científico y lo moral que se supone podría sustentar la vivencia de la sexualidad.

Según Giordan (1996) las concepciones que posee una persona se constituyen en una línea base para el análisis de la realidad, en una especie de decodificador que le permite comprender el mundo, afrontar nuevos problemas, interpretar situaciones inéditas, razonar para resolver una dificultad o responder de manera explicativa; también seleccionará las informaciones exteriores, las comprenderá y las integrará. Las concepciones se consideran como uno de los referentes más potentes para el desarrollo de la práctica pedagógica (Moreno, 2017).

Otras de las voces de los docentes expresan: "nosotros manejamos niños más pequeños, no se da la temática de derechos sexuales y reproductivos como tal, se aborda con ellos lo relacionado con identidad de género, no se habla de derecho sino cosas menos profundas"; esta manifestación devela la falta de claridad en la concepción de identidad de género, dado que es un concepto complejo, teniendo en cuenta que la identidad de género según las Naciones Unidas (sf), hace referencia a la vivencia interna e individual tal como cada persona la experimenta, la cual puede o no corresponder con el sexo asignado al momento del nacimiento, la complejidad de este 
concepto está dado desde la multidimensionalidad que expresa el mismo, si se tiene presente que incluye no solo la apariencia o la función corporal, sino, otras expresiones de género como la forma de vestir, el modo de hablar y las conductas.

Por lo anterior, se considera necesario valorar las prácticas de educación sexual desde una perspectiva de género en las instituciones escolares, con ello se lograría develar lo que en ellas se enseña sobre cómo deben ser hombres y mujeres, analizar los discursos de género que circulan en estas prácticas, verificar cómo son significadas, representadas, valoradas y ordenadas diversas identidades y cuáles hombres y mujeres son elegibles, legitimados/as, estigmatizados/as o marginados/ as (Quaresma da Silva, 2012).

\section{Vida Sexual}

La vida sexual abarca aspectos sociales, culturales e individuales, los cuales se encuentran íntimamente relacionados y mutuamente determinados, cada uno de ellos permite el desenvolvimiento y conductas del niño.

En la escuela es el docente quien se enfrenta con conductas que ameritan acompañamiento y preguntas urgidas de respuestas, acerca de esto una docente manifiesta "los niños presentan tantas inquietudes e interrogantes que nosotros como institución debemos orientarlos para que reconozcan sus derechos sexuales"; la escuela es el segundo ámbito de socialización e interacción, después del grupo familiar, en ella los niños continúan conformando su identidad en interacción con los demás (Cárdenas, 2015), es imperativo aunar fuerzas entre los dos actores familia y escuela, reflejado en la apertura de espacios de participación y apoyo de la familia, donde se generen ambientes cordiales entre ellos bajo una visión crítica y constructiva, con el interés común de fortalecer los procesos educativos relacionados con la educación sexual y los derechos sexuales y reproductivos de los niños y jóvenes.

La Familia, así como la Escuela son pilares fundamentales desde su campo de acción en la formación integral de los niños y jóvenes, deben propiciar canales de comunicación abiertos que generen formación integral desde el reconocimiento y respeto de los derechos sexuales del discente. El abordaje en temáticas formativas en salud sexual y reproductiva (motivación), brindan experiencias significativas al proceso formativo para los niños y adolescente, al convertirse en una oportunidad de ser protagonistas en la formación sobre su sexualidad, tanto en salud como reproducción, se da la oportunidad de reflexionar sobre ello para su propio conocimiento y para compartirlo con sus compañeros. (Cárdenas, 2015).

Cuando se habla sexualidad en la escuela las docentes responden "la verdad es que cuando los niños no conocen sus derechos son vulnerados verdad, la idea es que desde la institución, desde el proyecto y desde las diferentes actividades y áreas que se manejan en la institución se les brinde la posibilidad a los estudiantes de conocerlos para que ellos sepan cuáles son sus derechos, pero también cuáles son sus deberes y mirar que no puedan ser vulnerados en ningún momento, ni en la casa ni en la escuela, ni en ninguna parte" esto coincide con lo afirmado por Rodes (2012), en relación a que la infancia es una etapa del ciclo evolutivo donde el niño es frágil, indefenso lo que representa un riesgo significativo para que se vulneren sus derechos. Hay que tener en cuenta que en estos temas la familia juega un papel muy importante. Desde la escuela debe abordarse la sexualidad desde un enfoque de derechos y de promoción de la salud, esto hace que los niños y jóvenes se apropien más fácilmente de los mismos y tomen conciencia de ello (Mantilla, Oviedo \& Galvis, 2013). 
En consonancia con lo anterior, la sexualidad es concebida como parte integral del ser humano, se erige como esencia misma del individuo y así mismo se configura como un derecho, el cual se le relaciona con un conjunto importante de competencias ciudadanas y de ética social (Quaresma Da silva, 2012). La sexualidad le permitire, al individuo, acercarlo a lo que se conoce como desarrollo humano integral dentro de un plano emocional y afectivo.

En Colombia existe un entorno favorable en relación con el tema de sexualidad, ello se refleja en el Plan Nacional Decenal de Educación 20162026, en él se hace alusión entre otros aspectos a la diversidad sexual, sexualidad y educación sexual, es de resaltar el énfasis que se hace acerca de la promoción de la educación para la sexualidad desde una perspectiva de derechos que debe ser abordados en la escuela.

A partir de la existencia de una política orientada a la educación para la salud sexual se propone el desarrollo en las escuelas proyectos pedagógico como estrategias de inclusión con miras a contribuir a una educación integral y dar respuesta a las demandas que exige en la actualidad la Política.

El proyecto pedagógico es considerado como un proyecto participativo para hacer cotidiano el ejercicio de los derechos humanos sexuales y reproductivos, y una vivencia en la sexualidad que enriquezca el proyecto de vida de cada uno de los partícipes de la comunidad educativa (Cárdenas, M, 2015).

\section{Práctica Pedagógica}

La práctica pedagógica, es entendida como el campo de aplicación del discurso pedagógico que incluye el sujeto, la institución y un discurso quienes pueden aprehender, en la praxis, "los procesos de institucionalización" del saber pedagógico (Zuluaga, 1987,196-197) en consonancia con lo expuesto por Zuluaga, una de las docentes manifestó con relación a su práctica pedagógica lo siguiente: "pues particularmente yo les hablo y explico el por qué no deben hacer ciertas cosas, porque no beben tocarse ciertas partes a las niñas además utilizo dibujos, escritos y preguntas para reforzar sobre los derechos sexuales, todo esto de manera general y sencilla sin entrar en detalle"; esta manifestación del profesor deja ver una orientación limitada con relación a la profundidad y complejidad del tema de la sexualidad; además pone en evidencia la urgente necesidad de realizar inicialmente un proceso de capacitación a los docentes en educación sexual e involucrar en este proceso educativo a los diferentes profesionales, la comunidad y la familia (Rincón, 2016).

En Colombia, históricamente el abordaje pedagógico de la sexualidad se ha limitado a un enfoque biologicista, se concibe desde lo anatómico y fisiológico, reduciéndolo al sistema reproductor, con fines sólo procreativos. Para el abordaje de la educación sexual resulta fundamental que los docentes: reflexionen sobre sus propias experiencias escolares y familiares de educación sexual, conozcan cómo se desarrolla la sexualidad infantil y la identidad de género en edades tempranas, den cuenta de sus prácticas didácticas y pedagógicas con relación a Educación Sexual Integral (ESI), y articulen, interpreten y fundamenten los contenidos curriculares de educación sexual. Es Necesario enfocar la formación docente en educación sexual desde una Pedagogía del cuidado para respetar y poner en valor los derechos de los niños a educarse con libertad en relación con su sexualidad y su género, manifestar su afectividad, cuidando la salud propia y la de los otros (Gosende, 2016).

La formación sobre sexualidad, que asume el docente de hoy, está permeada por los rezagos de un sistema educativo anquilosado, 
punitivo y de represión de la sexualidad, característicos de épocas anteriores, en donde hablar se sexualidad era prohibido; ello influye y se refleja en una práctica pedagógica tímida y esquiva frente al tema (Santiago, 2016).

Lo anterior, motiva a ver la sexualidad, desde un enfoque complejo, concebida con un enfoque psicoanalítico fundamentada desde las siguientes perspectivas: 1) perspectiva de género, 2) el ejercicio de nuestros derechos; 3) perspectiva del cuidado del cuerpo y la salud; 4) valoración de la afectividad y 5) respeto por la diversidad. (Gosende, 2016).

\section{Acciones de promoción - Diagnóstico}

Para hablar de acciones de promoción se considera importante articularlas con un diagnóstico de las necesidades de formación docente orientado a la formulación de estrategias para la promoción de los D.S.R desarrolladas por los maestros y prepararse en el uso de la didáctica, con el fin de utilizar de una manera adecuada y pertinente los materiales y recursos pedagógicos. Con relación a este aspecto unos maestros expresaron: "aquí en la escuela y nosotros como profesores necesitamos identificar cuáles son nuestras falencias en este aspecto de DSR para poder trabajar en ello y poder brindar una orientación adecuada a nuestros chicos, con una metodología pertinente (...) sobre todo si tenemos en cuenta como está la situación o el tema en estos momentos en nuestras escuelas". Lo anterior guarda correspondencia con lo planteado por Mantilla, Hakspiel, Rincón, Smith \& Hernández (2012), al poner de manifiesto que el personal docente en Colombia reconoce la importancia de los procesos de educación para la sexualidad en el ámbito escolar, como una forma de promover la salud, y saber de su obligatoriedad conforme a la legislación vigente que rige el sector educativo, la mayoría se declara sin la suficiente formación para abordar dichos procesos. Saben que es importante hacerlo, ma- nifiestan interés en trabajar en esa área, pero se sienten inseguros (as) y sin las herramientas pedagógicas y didácticas para hacerlo bien.

Las necesidades de formación docente constituyen carencias en el desarrollo profesional del profesorado que varían en dependencia tanto de las exigencias sociales como de las particularidades individuales del profesorado y del estudiantado (González, T y González, M (2007). Estos vacíos de formación se reflejan en la ausencia del desarrollo de una estrategia educativa, dentro de su praxis, orientada a fomentar una concepción responsable sobre salud sexual y reproductiva en los estudiantes y favorecer la autorregulación de la salud sexual y reproductiva en los niños, adolescentes y jóvenes (Rodríguez, Sanabria, Contreras, Perdomo, 2013).

\section{Transversalidad}

El sistema educativo está llamado a promover cambios significativos, para contribuir a la formación de individuos capaces de convivir en una sociedad donde se desenvuelvan en forma tolerante, solidaria, honesta y justa. Dentro de este Sistema, los modelos curriculares suelen fundamentarse en la transversalidad o ejes transversales, con el fin de proporcionar elementos para la transformación de la educación. Los ejes transversales permiten establecer una articulación entre la educación fundamentada en las disciplinas del saber, los temas y las asignaturas de los planes de estudios para formar personas integras.

Integrar en la práctica pedagógica los saberes (ser, hacer, saber, convivir ) relacionados con los derechos humanos, de manera transversal en el currículo, se constituye en un aspecto fundamental que tributa a una formación ecuánime de la personalidad y contribuye a la resolución de problemas presentes en la sociedad, como es el caso particular de los DSR los cuales son violentados y vulnerados 
sobre todo en los niños y niñas, es allí donde el maestro debe involucrar en su quehacer pedagógico la enseñanza y práctica de los D.S.R desde la asignatura o área de conocimiento que desarrolle dentro del plan de estudio (Botero, 2008).

En contraste con lo anterior, una de las maestras refiere: "para que se de la transversalización esos hilos conductores que tienen los D.S.R y deben unirlos con los estándares y las competencias que nos permitirían involucrarlos en todas las áreas". Esta aseveración coincide con la de Reyes (2008), quien considera importante en el proceso formativo atender competencias, capacidades no sólo intelectuales sino también sus capacidades afectivas, motrices, de relación interpersonal, de inserción y de actuación social; es decir se aborde la enseñanza de manera multidimensional y articulada teniendo en cuenta el contexto, lo social, cultural, lo político y normativo.

\section{Identificación de las Acciones de Promoción de los D.S.R Desarrolladas por los Maestros de Básica Primaria de La I.E.}

En relación con las acciones de promoción de los D.S.R que desarrollan los maestros de básica primaria de la I.E rural; ellos coinciden que para desplegar acciones de promoción concretas necesitan de capacitación sobre el tema, esto se evidencia cuando manifiestan: "más que acciones yo diría....hay cosas que se nos salen de las manos y no las podemos manejar, entonces sería bueno, que nos capacitaran y miráramos de qué manera se podían solucionar, porque a veces uno peca y es por eso porque uno no sabe."

En consecuencia, se hace necesario que los maestros desde sus aulas de clases impartan acciones que les permitan hacer un abordaje transversal de situaciones relacionadas con los D.S.R; para lo cual es necesario: primero, la formación de los maestros en los componen- tes constitutivos, que abarcan el campo de los derechos, el respeto por las diferencias y el desarrollo de una sexualidad libre y responsable. Segundo, un cambio en la mirada en cuanto a la forma de abordar las practicas pedagógicas, las cuales deben diseñarse a partir de acciones basadas en procesos investigativos, con el ánimo de convertir el empoderamiento de los D.S.R en un proceso permanente y sistemático de formación que permita el crecimiento de los estudiantes y de los maestros, como persona humana consientes de la realidad que se vive en los entornos sociales y culturales.

\section{Propuesta Metodológica}

Luego de caracterizar las concepciones de los maestros acerca de los D.S.R. y de haber identificado las acciones de promoción desarrolladas por ellos, se realizó la configuración de los elementos de una propuesta metodológica para el abordaje transversal de los D.S.R. teniendo en cuenta la complejidad del ser humano como sujeto y objeto de la sexualidad, sujeto, porque lleva consigo sus valores, creencias, identidad, conocimiento y actitudes y objeto, porque en algunas situaciones es vulnerable y se atropellan sus derechos sexuales.

Se identificaron los elementos de la propuesta con el ánimo, de aportar a una transformación significativa y a la formación integral del ser humano, donde el sentido de formarse se conciba desde la integralidad y no desde aspectos particulares y fraccionados. La integralidad implica entonces que la práctica pedagógica debe ser asumida como un proyecto de inclusión educativa el cual nos conlleve a reflexionar sobre diferentes conceptos entre los cuales está la diversidad. Estos elementos son:

Cualificación de docentes en el componente de los derechos sexuales y reproductivos, a través de políticas institucionales, para la trans- 
formación de la práctica y la asuman como un proyecto investigativo en el cual se prioricen criterios relacionados con: la inclusión con el aprendizaje, la educación sexual integral y la construcción de nuevas ciudadanías.

Caracterización de los estudiantes (aspectos sociales, económicos, culturales y religiosos), a partir de este análisis de realidades contribuirá a la articulación de una manera más eficiente y eficaz los contenidos de enseñanza y de aprendizaje, para permitir posteriormente dar respuesta a las necesidades del educando.

Articulación al Proyecto Educativo Institucional (PEl) a un proyecto de educación sexual donde se evidencie el abordaje de los derechos sexuales y reproductivos como eje transversal en los procesos formativos, esto implica la resignificación del PEI, a luz de la reflexión crítica y al análisis de situaciones reales del contexto haciendo énfasis en la formación de la persona humana capaz de reconocerse como sujeto activo titular de sus derechos sexuales y reproductivos, con el cual desarrolle competencias orientadas al respeto de sí mismo, lo que implica aceptar su propia realidad y aceptarla (Rodríguez-Burgo \& García , M, 2017), el respeto hacia los demásy hacia su entorno.

Definición de estrategias que permitan hacer seguimiento a las acciones que den cuenta del trabajo interdisciplinar en relación con los derechos humanos, sexuales y reproductivos, se hace imperante la articulación entre los estándares de competencias de las diferentes áreas y aquellos lineamientos relacionados con el campo de la sexualidad, esto lleva a replantear el modelo de planeación curricular y a mirar la interdisciplinariedad como estrategia investigativa que permite el dialogo de saberes entre diferentes áreas se convierte en una alternativa para garantizar el abordaje transversal relacionado con el universo de los derechos sexuales y reproductivo.
Diseño de una política de evaluación que permita establecer criterios de efectividad para valorar el impacto en cuanto a las estrategias definidas, esta debe ser alineada a la propuesta curricular que adopte la institución educativa para su respectivo funcionamiento. Esta política, debe estar sustentada en un enfoque investigativo cualitativo etnográfico, con el propósito de hacerle seguimiento de manera simultánea al desarrollo de habilidades y del aprendizaje significativo de los estudiantes, en coherencia con las características particulares de los entornos sociales y culturales que rodean a cada uno de ellos.

\section{Conclusiones o Consideraciones Finales}

Los resultados obtenidos permitieron establecer algunas conclusiones que coinciden con lo descrito en estudios similares en relación con la escasa apropiación y conocimiento que tienen las docentes al momento de impartir sobre sus derechos sexuales y reproductivos, así como el de expresar ciertos términos por el tabú que aún existe en algunas de ellas.

Los docentes reconocen que no poseen formación sobre cómo abordar los derechos sexuales y reproductivos desde su práctica pedagógica, se demostró un discurso poco elaborado (escueto) y basado en ideas generales al momento de abordar aspectos relacionados con el tema.

Se develó que en la educación sexual circulan creencias sobre género y sexualidad que fundamentan la forma cómo se ha instrumentalizado la formación en educación sexual desde conceptos, creencias y concepciones hegemónicas de masculinidad y feminidad, así como desde lo biológico y reproductivo; esta visión paradigmática obstaculiza el avance en la transversalización de esta área en el currículo.

Los profesores reconocen la necesidad de ayu- 
da de personal capacitado al momento de impartir o compartir conocimiento sobre DSR, y de continuar gestando en todas las instituciones educativas, con el apoyo de las entidades comprometidas, programas de educación sexual, según el nivel educativo, igualmente, se requieren procesos de apoderamiento en los grupos de niños y jóvenes para que de esta manera puedan alcanzar bienestar, acceso, toma de conciencia, participación y control y así crear nuevas visiones de la salud y de la vida.

El desconocimiento de los DSR por parte del estudiante los hace vulnerable, en ocasiones inseguro, el docente le resta trascendencia a la falta de formación cuando se trata de escolares menores de 10 años, lo que expone al menor a que se le puedan vulnerar sus derechos.

Por consiguiente, las actividades que desarrollan los maestros en relación con la a promoción de los D.S.R, son pocas, y a la vez aisladas del ejerció de su práctica pedagógica. Lo anterior indica la necesidad de impartir procesos de formación desde el universo de los D.S.R, es urgente que desde sus áreas del conocimiento sean articulados como saberes transversales, y de esta forma garantizar el empoderamiento por parte de la comunidad educativa en cuanto a la existencia de estos derechos, como parte fundamental de la formación desde una perspectiva humanística.

En tal sentido se determina que los D.S.R, deben asumirse desde una perspectiva transversal, haciendo un proceso de articulación a las áreas del conocimiento a partir de las diferentes planeaciones curriculares; en otro sentido se considera fundamental que las maestras sean cualificadas desde el punto de vista de la didáctica, con el fin de hacer uno adecuado y pertinente uso de los materiales y recursos pedagógicos. En otro sentido consideran importante también buscar alternativas que conlleven a involucrar a los padres y madres de familia en estos procesos, con el objetivo que todos los actores que hacen parte del sistema educativo se comprometan de forma decidida.

El diseño e implementación de estrategias educativas es fundamental para fomentar en los niños el pensamiento autorregulado y responsable sobre salud sexual y reproductiva, lo cual contribuirá a favorecer el autocontrol de su salud sexual y reproductiva. 


\section{Referencias}

Basto-Torrado, S. P. (2011). De las concepciones a las prácticas pedagógicas de un grupo de profesores universitarios. Magis. Revista Internacional de Investigación en Educación, 3(6). 393-412

Botero, Carlos Alberto (2008). Los Ejes Transversales como instrumento pedagógico para la formación de valores. Revista Iberoamericana de Educación. 45 (2). Disponible en: http://mww.rieoei.org/ deloslectores/2098Botero.pd

Cárdenas, M, J. (2015). La educación sexual como estrategia de inclusión en la formación integral del adolescente. Praxis. Vol. 11, 103 - 115

Cortez Quevedo, K., Fuentes Quelin, V., Villablanca Ortiz, I., \& Guzmán, C. (2013). Creencias docentes de profesores ejemplares y su incidencia en las prácticas pedagógicas. Estudios pedagógicos (Valdivia), 39(2), 97-113.

García, P. A. R., \& González, A. D. P. O. (2017). Problematización de la Educación Sexual: Reflexiones Acerca de la Sexualidad En La Escuela Colombiana. Revista Bio-grafía Escritos sobre la biología y su enseñanza, 9(16), 23-29.

doi: $\quad$ http://dx.doi.org/10.17227/20271034.vol.9num.16biografia23.29

Giordan, A. (1996). ¿Cómo ir más allá de los modelos constructivistas? La utilización didáctica de las concepciones de los estudiantes. Revista Investigación en la Escuela, (28), 7-22.

Giraldo Neira, O. (2006): "La educación sexual: el aprendizaje desde la cuna". En Revista Javeriana. 721: 28. Enero-febrero. En: República de Colombia, Ministerio de Educación Nacional, Módulo 1.

González Tirados, R. y González Maura, V. (2007): «Diagnóstico de necesidades y estrategias de formación docente en las universidades», en Revista Iberoamericana de Educación, 43 (6).

González Martínez, M. N., Vásquez De la Hoz, F., Campo Ternera, L., Miranda Medina, C. F., Silvera Sarmiento, A., Agudelo Gómez, C. J., \& Parra Vega, J. A. (2014). Estilos de enseñanza y docencia Universitaria. Ediciones Universidad Simón Bolívar.

Gosende, E. E. (2016). Educación Sexual Integral en el nivel inicial: Experiencias de Capacitación Docente desde una Pedagogía del Cuidado. In VIII Congreso Internacional de Investigación y Práctica Profesional en Psicología XXIII Jornadas de Investigación de la Facultad de Psicología XII Encuentro de Investigadores (No. 1, p. 139).

Izquierdo Hernández, A. (2013). Salud sexual y reproductiva. Revista Cubana de Enfermería, 29(1), 1-2.

Mantilla Uribe, B. P., Oviedo Cáceres, M. D. P., \& Galvis Padilla, D. C. (2013). Programas de educación sexual y reproductiva: significados asignados por jóvenes de cuatro municipios de Santander, Colombia. Revista Hacia la Promoción de la salud, 18(1).54

Mantilla Uribe, B., \& Hakspiel Plata, M., \& Rincón Méndez, A., \& Smith Hernández, D., \& Hernández Quirama, A. (2012). Promoción de derechos sexuales y reproductivos en adolescentes de Bucaramanga, Floridablanca y Lebrija - Colombia. Revista de la Universidad Industrial de Santander. Salud, 44 (3), 13-23

Ministerio de la Protección Social. (2003). Política Nacional de Salud Sexual y Reproductiva. Recuperado de https://www.minsalud.gov.co/Documentos\%20 y\%20 Publicaciones/POL\% C3\% 8DTICA\%20 NACIONAL\%2ODE\%2OSALUD\%2OSEXUAL\%20Y\%20 REPRODUCTIVA.pdf

Moraes, R; Galiazzi, Do Carmo.M. (2007). Análisis Textual Discursivoljuí: Unijuí.

Moreno, E. A. R. (2017). Concepciones de práctica pedagógica. Revista Folios, (16), 105-129.

Plan Nacional Decenal de Educación (PNDE) 2016-2026. Ministerio de Educación Nacional - MEN. Disponible en: http://www.mineducacion.gov.co/1621/articles 312490_archivo_pdf_plan_decenal.pdf

Preinfalk-Fernández, M. L. (2015). Desafíos de la formación docente en materia de educación sexual. Revista Electrónica Educare, 19(1)

Quaresma da Silva, Denise. (2012). The production of normal and abnormal: a study on gender and sexuality beliefs among teachers of public schools in Novo Hamburgo/Brazil. Subjetividad y procesos cognitivos, 16(1), 178-199.

Ramírez Durán, G., \& Barriento García, M. (2015). Salud sexual y reproductiva. Carta al Editor, Revista Cubana de Enfermería, 31(1) 
Reyes, R. (2008). Evaluación de la docencia en el nivel superior del estado de Tlaxcala. En M. Rueda (Coord.), ¿Es posible evaluar la docencia en la universidad? Experiencias en México, Canadá, Francia, España y Brasil (pp. 195-208). México: UABJO-ANUIES.

Rincón, C. A. F. (2016). Yo/nosotros en harmonía con tu/ otros y el cuidado transpersonal-humanizado en sexualidad. Libros Editorial UNIMAR

Ríos, A. L. (2008). Alcance de las políticas públicas en el área de salud sexual y reproductiva dirigidas a los adolescentes en Colombia. Salud Uninorte, 24(2).

Rodes, LLoret Fernando. (2012) Vulnerabilidad infantil. Ediciones Díaz de santos. España.

Rodríguez-Burgos, K., \& García, M. H. (2017). Respeto y tolerancia como valores promotores de la Cultura de Paz en padres de niños en edad preescolar en el municipio de San Nicolás de los Garza, Nuevo León, México. Revista Pensamiento Americano, 10(18).

Rodríguez Cabrera, Aida, Sanabria Ramos, Giselda, Contreras Palú, Maria Elena, \& Perdomo Cáceres, Belkis. (2013). Educational strategy on sexual and reproductive health promotion aimed at adolescents and university young people. Revista Cubana de Salud Pública, 39(1), 161-174.

Rodríguez, K. \& Hinojosa, M. (2017). Respeto y tolerancia como valores promotores de la Cultura de Paz en padres de niños en edad preescolar en el municipio de San Nicolás de los Garza, Nuevo León, México. Pensamiento Americano, 10(18), 96-114.

Santiago, J. (2016). Otro discurso pedagógico y didáctico sobre la enseñanza de la geografía en el trabajo escolar cotidiano. Pensamiento Americano, 9(16), 171-188.

Santos, A., Baquero, P., Molano, M. \& Pardo, A. (2006) Prácticas pedagógicas universitarias: aproximaciones para su comprensión. Bogotá: Universidad de la Salle.

Vásquez, L. E. (2006). “Informe práctica pedagógica investigativa y proyección social" Universidad de la Salle. Edit Universitario. Bogotá D.C.

Velis, F. V. (2017). Educación sexual y diversidad en los programas educativos de América Latina. Revista Electrónica Diálogos Educativos, 10 (20), 75-104.

Zuluaga Garcés, O. (1987). Pedagogía e historia. Bogotá: Editorial Universidad de Antioquia.
2018, Vol. 11(21) 237-252 @The Author(s) 2018 Reprints and permission: www.americana.edu.co

https://www.coruniamericana.edu.co/publicaciones/ojs/index.php/pensamientoamericano/index 